\title{
A genomic glimpse of aminoacyl-tRNA synthetases in malaria
} parasite Plasmodium falciparum

\author{
Tarun Kumar Bhatt ${ }^{1}$, Charu Kapil1,2, Sameena Khan ${ }^{1,3}$, \\ Mohamad Aman Jairajpuri², Vinay Sharma ${ }^{3}$, Daniele Santoni ${ }^{4,5}$, \\ Francesco Silvestrini ${ }^{5}$, Elisabetta Pizzi ${ }^{5}$ and Amit Sharma*1
}

Address: ${ }^{1}$ Structural and Computational Biology Group, International Centre for Genetic Engineering and Biotechnology, New Delhi 110 067, India, ${ }^{2}$ Department of Biosciences, Jamia Millia Islamia University, Jamia Nagar, New-Delhi 110025 , India, ${ }^{3}$ Department of Bioscience and Biotechnology, Banasthali Vidyapith University, Banasthali, Rajasthan 304 022, India, ${ }^{4}$ Barcelona Institute for Research in Biomedicine, Barcelona Science Park, C/Samitier 1-5, Barcelona 08015, Catalonia, Spain and ${ }^{5}$ Dipartimento di Malattie Infettive, Parassitarie ed Immunomediate, Istituto Superiore di Sanità, Viale Regina Elena, 299, 00161 Rome, Italy

Email: Tarun Kumar Bhatt - tkbhatt@icgeb.res.in; Charu Kapil - charu@icgeb.res.in; Sameena Khan - sameena@icgeb.res.in; Mohamad Aman Jairajpuri - m_jairajpuri.bi@jmi.ac.in; Vinay Sharma - vinaysharma30@yahoo.co.uk;

Daniele Santoni - daniele.santoni@irbbarcelona.org; Francesco Silvestrini - francesco.silvestrini@iss.it; Elisabetta Pizzi - elisabetta.pizzi@iss.it; Amit Sharma* - amit.icgeb@gmail.com

* Corresponding author

Published: 31 December 2009

BMC Genomics 2009, 10:644 doi:10.1 186/|47|-2/64-10-644
Received: 3 July 2009

Accepted: 31 December 2009

This article is available from: http://www.biomedcentral.com/I47I-2/64/I0/644

(c) 2009 Bhatt et al; licensee BioMed Central Ltd.

This is an Open Access article distributed under the terms of the Creative Commons Attribution License (http://creativecommons.org/licenses/by/2.0), which permits unrestricted use, distribution, and reproduction in any medium, provided the original work is properly cited.

\begin{abstract}
Background: Plasmodium parasites are causative agents of malaria which affects $>500$ million people and claims $\sim 2$ million lives annually. The completion of Plasmodium genome sequencing and availability of PlasmoDB database has provided a platform for systematic study of parasite genome. Aminoacyl-tRNA synthetases (aaRSs) are pivotal enzymes for protein translation and other vital cellular processes. We report an extensive analysis of the Plasmodium falciparum genome to identify and classify aaRSs in this organism.

Results: Using various computational and bioinformatics tools, we have identified 37 aaRSs in $P$. falciparum. Our key observations are: (i) fraction of proteome dedicated to aaRSs in $P$. falciparum is very high compared to many other organisms; (ii) 23 out of 37 Pf-aaRS sequences contain signal peptides possibly directing them to different cellular organelles; (iii) expression profiles of Pf-aaRSs vary considerably at various life cycle stages of the parasite; (iv) several PfaaRSs posses very unusual domain architectures; ( $v$ ) phylogenetic analyses reveal evolutionary relatedness of several parasite $a a R S s$ to bacterial and plants aaRSs; (vi) three dimensional structural modelling has provided insights which could be exploited in inhibitor discovery against parasite aaRSs.

Conclusion: We have identified 37 Pf-aaRSs based on our bioinformatics analysis. Our data reveal several unique attributes in this protein family. We have annotated all 37 Pf-aaRSs based on predicted localization, phylogenetics, domain architectures and their overall protein expression profiles. The sets of distinct features elaborated in this work will provide a platform for experimental dissection of this family of enzymes, possibly for the discovery of novel drugs against malaria.
\end{abstract}




\section{Background}

Aminoacylation is the process of adding an aminoacyl group to the 3' end (CCA) of the tRNA molecule. tRNA is aminoacylated with a specific amino acid by aminoacyltRNA synthetase (aaRSs). aaRSs are responsible for attaching correct amino acid onto the cognate tRNA molecule in a two-step reaction. The amino acid is first activated with ATP forming an aminoacyladenylate intermediate. Once activated, this amino acid is transferred to the 3' end of its corresponding tRNA molecule to be processed during protein synthesis. All aaRSs require divalent cation $\mathrm{MgCl}_{2}$ for their aminoacylation reaction $[1,2]$.

\section{Reaction:}

1. amino acid + ATP $\rightarrow$ aminoacyl-AMP + PPi

2. aminoacyl-AMP + tRNA $\rightarrow$ aminoacyl-tRNA + AMP

The aaRSs are divided into two major classes based on structural topology of their active sites. Class I aaRSs represent 11 amino acids, including Arg, Cys, Gln, Glu, Ile, Leu, Lys, Met, Val, Trp and Tyr. Class II aaRSs includes 10 amino acids - Ala, Asp, Asn, Gly, His, Lys, Phe, Pro, Ser and Thr. Core domains of class I enzymes are characterized by a Rossmann fold which consists of $\alpha$-helices and $\beta$-pleated sheets. This domain contains two conserved motifs ('HIGH' and 'KMSKS') which are directly involved in ATP binding. Catalytic domain of class II enzymes has a unique fold with a central core of anti-parallel $\beta$ strands flanked by $\alpha$ helices [3]. There are three weakly conserved motifs, two of them are involved in ATP binding while the third one plays a role in homo dimerization. Class I enzymes bind ATP in an extended conformation while class II do so in a bent conformation. The two aaRS classes have different modes of aminoacylation - class I enzymes aminoacylate the 2'OH of the cognate tRNA whereas class II enzymes aminoacylate 3'OH of the tRNA (with the exception of PheRS) [4]. All known aaRSs are multidomain proteins with complex modular architectures [5]. In addition, eukaryotic aaRSs are distinguished by the presence of appended domains at either the $\mathrm{N}$ - or C-terminus which are generally absent from their bacterial/archaeal counterparts [6]. These appendages to the catalytic cores of several aaRSs are non-catalytic and instead function to mediate protein- protein interactions or act as general RNA-binding domains [7-9].

In mammalian cells, some aaRSs are present as a larger multi- aaRS complex (MSC) composed of nine synthetases (arginyl-, aspartyl-, glutamyl-, glutaminyl-, leucyl-, lysyl-, isoleucyl-, methionyl- and prolyl-tRNA synthetases) [10-12]. The MSC is composed of a mixture of class I and class II aaRSs along with three non- aaRS proteins p38, p43 and p18. It is not clear why certain
aaRSs exist as a complex while some are in free form. MSC might help in efficient protein synthesis by preventing mixing of charged tRNAs with cellular pool and by increasing local concentration of tRNA near the site of protein synthesis [13].

The accuracy of tRNA aminoacylation reaction is critical in ensuring fidelity in protein translation [14]. To achieve this accuracy, some aaRS enzymes possess a proofreading (editing) mechanism that hydrolyzes tRNAs aminoacylated with the non-cognate amino acid [15]. For example, editing domains may be found attached to alanyltRNA synthetase (AlaRS), leucyltRNA synthetase (LeuRS) and so on [16-21]. In other cases, the editing domain is not attached to aaRS but rather functions as an individual protein [22,23]. For example, YbaK protein from Haemophilus influenza is capable of efficiently editing Cys-tRN$\mathrm{A}^{\text {Pro }}$ [24]. ThrRS has been shown to have another editing domain called NTD which can cleave the bond between D-amino acid and tRNA [25].

Recently it has been shown that aaRSs are not only involved in protein synthesis but also perform many noncatalytic and non-canonical roles in RNA processing/trafficking, apoptosis, rRNA synthesis, angiogenesis and inflammation [26-30]. These versatile properties of aaRSs are the outcome of their differential cellular localization, nucleic acid binding properties, protein-protein interactions and collaboration (fusion) with additional domains. In case of malaria parasite, apicoplast proteins and pathways have already received particular attention as drug targets [31]. In this work we present a study of aaRSs from $P$. falciparum - the most virulent agent of human malaria. Our aim for this study was to use bioinformatics tools to (a) discover special and unusual modules present in parasite aaRSs which are potentially absent from human homologues, and (b) to identify potential new drug targets based on this protein family.

\section{Results and Discussion}

\section{Sequence extraction and analysis}

We exploited current annotation available in PlasmoDB [32] to identify the repertoire of aaRSs in P. falciparum genome. According to Enzyme Commission (EC) 37 proteins in PlasmoDB (see additional file 1) are annotated as belonging to the EC group 6.1.1. (EC number provided for aaRSs). Although in many cases current annotations allow an assignment to Class I or II of aaRSs, for some annotations are still preliminary. Due to this, we used Hidden Markov Models (HMMs) for identifying aaRSs in $P$. falciparum. For each aaRS a set of known sequences was utilized to construct $20 \mathrm{HMMs}$ (see methods for details). For each database search a score distribution was obtained and 4 cutoffs were considered to identify aaRS. Results are reported in Table 1 . We observed that 2 proteins anno- 
Table I: Results of database searches by HMM models of aaRS@.

\begin{tabular}{|c|c|c|c|c|c|c|c|c|c|c|}
\hline cutoff & HMMAla & HMMArg & HMMAsn & HMMAsp & HMMCys & HMMGIn & HMMGlu & HMMGly & HMMHis & HMMlle \\
\hline$c>50$ & PFI3_0354 & PFL0900c & PFE0475w* & PFB0525w* & PFI0_0I49 & PFI3_0I70 & MALI3PI.28I & PFI4_0198 & PFI4_0428 & PFI3_0I79 \\
\hline $20<c<50$ & & & PFB0525w* & $\begin{array}{l}\text { PFA0145c } \\
\text { PFE0475w* }\end{array}$ & & PFI3_0257* & PFI3_0257* & & PFII645c & \\
\hline $10<c<20$ & & & PFE07I5w* & & & & & & & \\
\hline $5<c<10$ & & PFI0680c & & PFE07I $5 w^{*}$ & & & & & & PFLI210w \\
\hline Cutoff & HMMLeu & HMMLys & HMMMet & HMMPhe & HMMPro & HMMSer & HMMThr & HMMTrp & HMMTyr & HMMVal \\
\hline$c>50$ & PF08_00II & PFI3_0262 & PFI0_0340 & PFA0480w & PFL0670c & PF07_0073 & PFII_0270 & PFI3_0205 & $\begin{array}{c}\text { MAL8PI.I2 } \\
5\end{array}$ & PFI4_0589 \\
\hline $20<c<50$ & PFFI095w & PFI4_0166 & PFI0_0053 & PFFOI80w & PFII $240 c$ & PFL0770w & & PFL2485c & PFII_018I & \\
\hline $10<c<20$ & & & & $\begin{array}{l}\text { PFLI540c } \\
\text { PFII_005I }\end{array}$ & & & & & & \\
\hline
\end{tabular}

$5<c<10$

* hits with more than one assignment to HMM model.

@Two proteins PFI4_040I and PFC0470w were not found by HMMs which were annotated as tRNA binding protein and ValRS in PlasmoDB respectively.

tated as belonging to EC group 6.1.1.- in PlasmoDB are not found by HMMs - PF14_0401 annotated as MetRS is instead a generic tRNA binding protein as elucidated in the genome re-annotation process, while the second one (PFC0470w) is still mis-annotated as ValRS. A total of 18 $P f$-aaRSs can be classified within the 10 aaRSs that define class I. All members of this class are represented in the $P$. falciparum proteome. The annotations of these sequences are summarized in additional file 1 . Similar to class I Pf$a a R S s$, the class II $P f$-aaRSs have a total of 18 sequences for 10 different amino acid synthetases. Four genes are present in $P$. falciparum for PheRS but these likely encode for 1 heterodimeric and 2 monomeric versions of PheRS.

In order to carry out comparative analyses of aaRSs of $P$. falciparum with those of other species we considered aaRS sequences from several organisms representing three domains of life (see methods section). As expected, we found variable number of aaRSs in different species. $M$. jannaschii (archaebacteria) and M. tuberculosis (bacteria) have the lowest aaRSs count amongst other organisms like E. coli, S. cerevisiae, D. discoidium, P. falciparum, O. sativa, $R$. norvegicus, D. melanogaster, and $H$. sapiens. Human bears the highest number of aaRSs in this analysis (Figure 1a). Our analysis also shows that $P$. falciparum has the highest aaRS fraction (relative to its proteome size) when compared with bacteria, yeast and human counterparts (Figure 1b). The number of individual aaRS varies in different species. For example, when individual aaRSs from human and $P$. falciparum were compared it was evident that AlaRS and ThrRS were higher in number in humans (Figure 2). Presence of more than one copy of each aaRS in an organism may indicate additional biological, temporal or spatial roles for these enzymes as several aaRSs also perform non-canonical functions [33]. In this work we describe in detail the 37 Pf-aaRSs.

\section{Indirect pathways of aminoacylation}

It was earlier believed that $20 a a R S s$ were necessary for the incorporation of 20 amino acids in proteins. But surprisingly, some archaea, bacteria and chloroplasts lack GlnRS and AsnRS enzymes [34-38]. Interestingly, these organisms use an alternate pathway based on tRNA dependent amino acid transformation. A non-discriminating GluRS charges tRNA Gln with glutamic amino acid and then a second enzyme called tRNA-dependent amidotransferase (AdT) amidates glutamate to make glutamine. A corresponding reaction occurs in case of asparagine residues. In case of $P$. falciparum, occurrence of glutamate-tRNA synthetase (PF13_0257, MAL13P1.281) and amidotransferase subunit A (PFD0780w) \& subunit B (PFF1395c) together indicates presence of both direct and indirect pathways for aminoacylation $[39,40]$. Both subunits of amidotransferase have apicoplast targeting signals suggesting an indirect pathway for aminoacylation in P. falciparum apicoplast. The expression of Pf-AdT subunit A is predicted in all life cycle stages of parasite based on proteomic and microarray data. We therefore feel that this 
a)

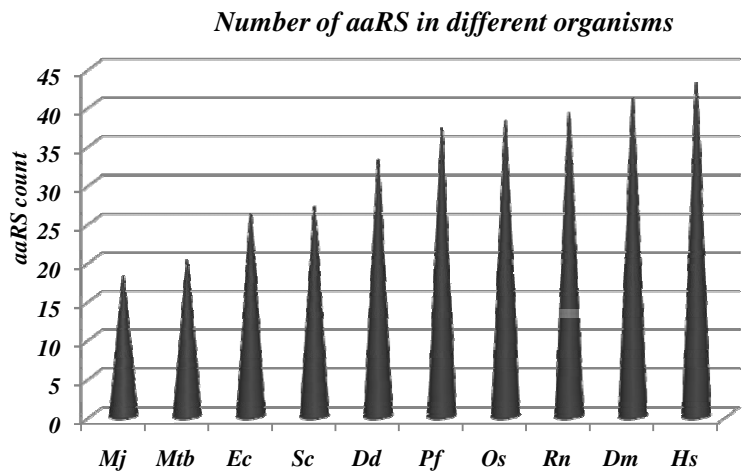

b)

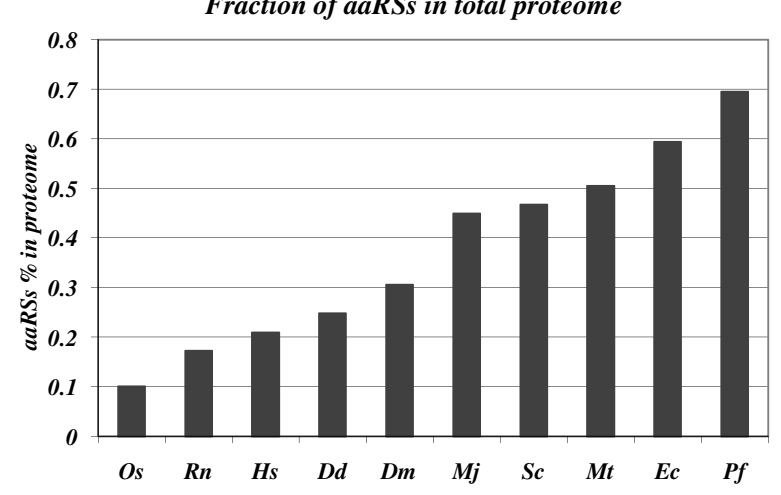

Figure I

(a) Predictied number of aaRSs present in Plasmodium falciparum (Pf), Rattus norvegicus (Rn), Saccharomyces cerevisiae (Sc), Drosophila melanogaster (Dm), Homo sapiens (Hs), Oryza sativa (Os), Dictyostelium discoidium (Dd), Mycobacterium tuberculosis (Mtb), Escherichia coli (Ec), and Methanocalclococcus jannaschii (Mj). (b) Diagram representing fraction of proteome (in percentage) dedicated to the aaRS proteins in various organisms.

pathway must also be active in the parasite apicoplast. We could not find sequence homologues of enzymes involved in indirect aminoacylation of cysteine residues [41-43] in the proteome of $P$. falciparum.

\section{The multi-synthetase complex (MSC)}

In mammalian cells, some aaRSs are present as a larger multi-aaRS complex (MSC). A constituent of the MSC protein p43 - has sequence homologue (PF14_0401 EMAP-II-like cytokine) in P. falciparum although there is no evidence for presence of MSC in malaria parasites. Interestingly, p43 is not only required for stability of the MSC complex but also functions as a proinflammatory cytokine [44-46]. Role of p43 homolog in P. falciparum is unknown, but evidence from other organisms indicates that MSC functions in protein stability, efficient protein translation and protein elongation [47]. Sequence identity between $P$. falciparum $\mathrm{p} 43$ and its human homolog is $\sim 24 \%$ and based on microarray data 443 seems to be expressed at asexual life cycle stages of $P$. falciparum. A mitochondrial targeting signal was also predicted for parasite p43 but the role of $\mathrm{p} 43$ in parasite remains to be explored experimentally.

\section{Targeting of aaRSs in the parasite}

aaRSs are not only involved in protein synthesis but also in various other cellular activities including intron splicing, translational regulation and tRNA channeling. Diversified roles for aaRSs necessitate their presence (transit) into various cellular compartments. We therefore analyzed $P$. falciparum aaRS sequences for presence of putative signal sequences predicted by MITOPROT, PredictNLS and PATS for mitochondria, nucleus and apicoplast respectively. We found that $23 P$. falciparum aaRSs have signal peptides, possibly for directing them to different cellular organelles. Another 14 aaRSs from P. falciparum may be resident in the parasite cytoplasm (Figure 3a). Apicoplast is known to have protein synthesis machinery which may use aaRSs [48]. Trafficking of nuclear encoded aaRSs to the apicoplast may explain why 20 out of 37 Pf-aaRSs have apicoplast targeting signals. Our data indicate that out of total $\sim 20$ Pf-aaRSs bearing apicoplast targeting signals, $\sim 12$ aaRSs may be exclusive to this organelle. Others are predicted to be shared between apicoplast, nucleus and mitochondria (Figure 3b). It has been earlier shown that some tRNAs need to be aminoacylated in the nucleus before they can be exported to the cytoplasm, an observation indicating occurrence of aminoacylation reaction (mediated by aaRSs) inside the nucleus [49]. In P. falciparum, we found 10 aaRSs with nuclear localization signals but only one is predicted to be exclusively resident in the nucleus (PFA0480w- PheRS). Interestingly, we found no Pf-aaRS sequences with specific PEXEL (Plasmodium export element) motifs. This motif is found in parasite proteins that are exported beyond the parasitophorous vacuole membrane [50,51].

\section{Expression profiles of $\mathrm{P}$. falciparum aaRSs}

In order to study expression of aaRS during life cycle of the malaria parasite, we took advantage of available transcriptomics and proteomics data from PLASMODB. Firstly, we analyzed proteomic data from several independent experiments and compared them with transcriptomics data by Le Roch [52]. The latter sets of data were obtained using the affimetrix technology and hence provide a quantitative measure of mRNA levels in the parasite. Our results are provided in Table 2. Interestingly, we found that mRNA levels of potential apicoplast proteins (AP in the table) are lower on average (mean $1=44.6$; mean $2=41.5$; gam $=91.3$; spor $=58.1)$ than those of potential cytoplasmic proteins $($ mean $1=259 ;$ mean $2=264.8$; gam $=174.8$; spor $=73.8)$. Proteomic data confirmed that while the cytoplasmic aaRS are found in almost all stages, the apicoplast aaRS are rarely found in the parasite. This could be in part due to experimental limits in the identification of 


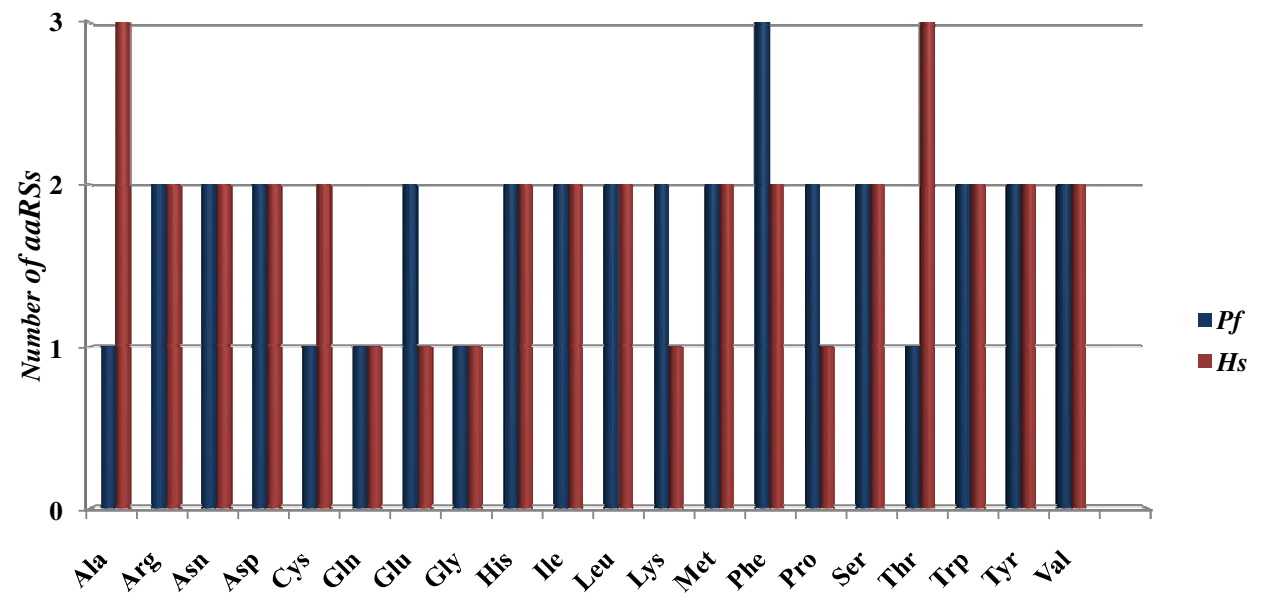

Figure 2

Bar graph showing number of different aaRSs in Plasmodium falciparum and Homo sapiens. The number of alanyland threonyl- tRNA synthetases is higher in humans whereas $P$. falciparum seems richer in phe tRNA synthetases.

apicoplast proteins by mass spectrometry. Indeed, when we carried out a chi-quadro test we found that proteins predicted to be targeted to apicoplast are significantly less represented $\left(\mathrm{p}<10^{-4}\right)$ in the sample of proteins identified by mass spectrometry. For these reasons we limited analysis of gene expression profiles only for putative cytoplasmic proteins. We considered trascriptomics data for sexual stages and asexual stages [52,53]. We considered a reduced set of the time course gene expression data (22 time points instead of 48) and normalized data by Le Roch (see methods for details). This allowed us to analyse the expression of aaRS genes along all the intra-erythrocytic life cycle of the parasite (Table 2). Further observations of the protein expression profiles indicated that some aaRSs were exclusively detected at specific stages like, LeuRS (PF08_0011) and AspRS (PFE0715w) in sporozoites; IleRS (PFL1210w), SerRS (PF07_0073), GlnRS (PF13_0170), HisRS (PF14_0428) and PheRS (PFA0480w) in merozoites; AsnRS (PFE0475w), PheRS (PF11_0051) and HisRS (PFI1645c) in trophozoites and TrpRS (PF13_0205) in gametocyte stages (Figure 4).

\section{Domain architecture of $\mathbf{P}$. falciparum aaRSs}

aaRSs are multi-domain proteins typically consisting of a conserved catalytic domain and an anti-codon binding domain. In addition, some aaRSs have RNA binding and editing domains that cleave incorrectly aminoacylated tRNA molecules [54]. Additional functional domains may be appended to aaRSs in the course of biological evolution $[55,56]$. Careful examination of 37 identified $P$. falciparum aaRSs using Pfam database showed that most of them have a generic modular architecture that adheres to prototypical aaRSs (Figure 5). The remaining P. falciparum aaRSs or related proteins like PF14_0423 (protein having serine-threonine kinase domain in fusion with an anti- codon binding module) have complex domain architectures. In several, concatenation of unusual domains such as Ybak, GST, Ser-Thr kinase and DNA binding domains is evident (Figure 5). The functional relevance of these additional domains fused to typical aaRS in $P$. falciparum needs to be experimentally addressed. Intriguingly, two of the four Pf-PheRS subunits contain DNA binding domains (PF11_0051, PFA0480w). It is likely that the PheRS, in addition to its aminoacylation function, influences other cellular processes via DNA binding [57]. Consistent with its potential DNA binding property, the $P$. falciparum PheRS (PFA0480w) has a nuclear localization signal. The CysRS of B. subtilis (which also contains a DNA binding domain) is believed to play a role in initiating chromosomal replication [58]. Therefore, functional roles for $P$. falciparum PheRSs may extend from aminoacylation to DNA recognition and replication - a suggestion that requires experimental investigation. Similarly, it has been shown that GST or GST homology domains can help in complex formation of aaRSs with multifunctional factors (p38, p18) [56,57]. Additional data show that deletion of GST homology domain from the C-terminal region of $\mathrm{p} 38$ results in the dissociation of EPRS (Glutamyl-prolyl-tRNA synthetase) and MetRS from the MSC complex [59]. Mammalian ValRS associated with elongation factor subunits also contain the GST homology domain [60-62]. Thus, the presence of GST domains might be a crucial feature of aaRSs. P. falciparum proteome has two such proteins with GST domains appended to MetRS (PF10_0340) and GluRS (PF13_0257). We also found a most interesting fusion of anticodon binding domain with a serine-threonine kinase (PF14_0423) in P. falciparum. This unusual kinase seems to be expressed throughout the life cycle of parasite (microarray data) and interestingly is predicted to be localized to the parasite nucleus. Clearly, the presence 
a)

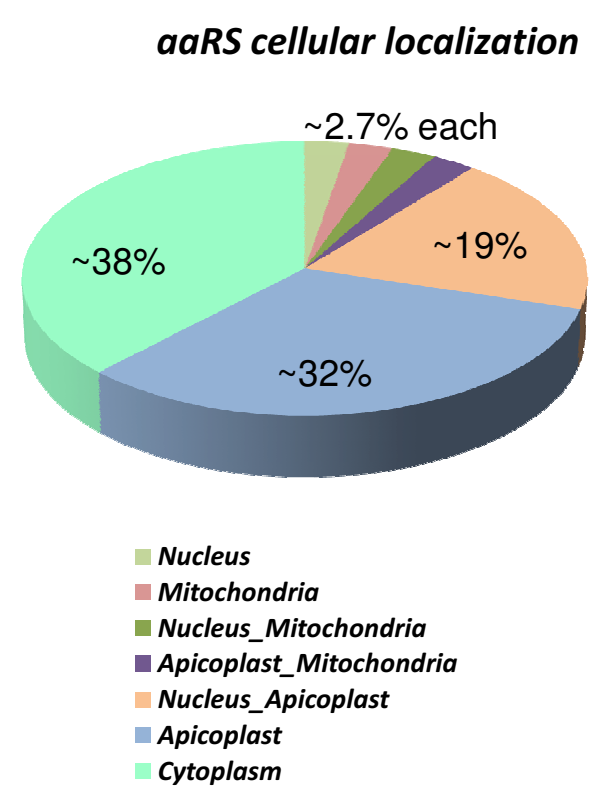

b)

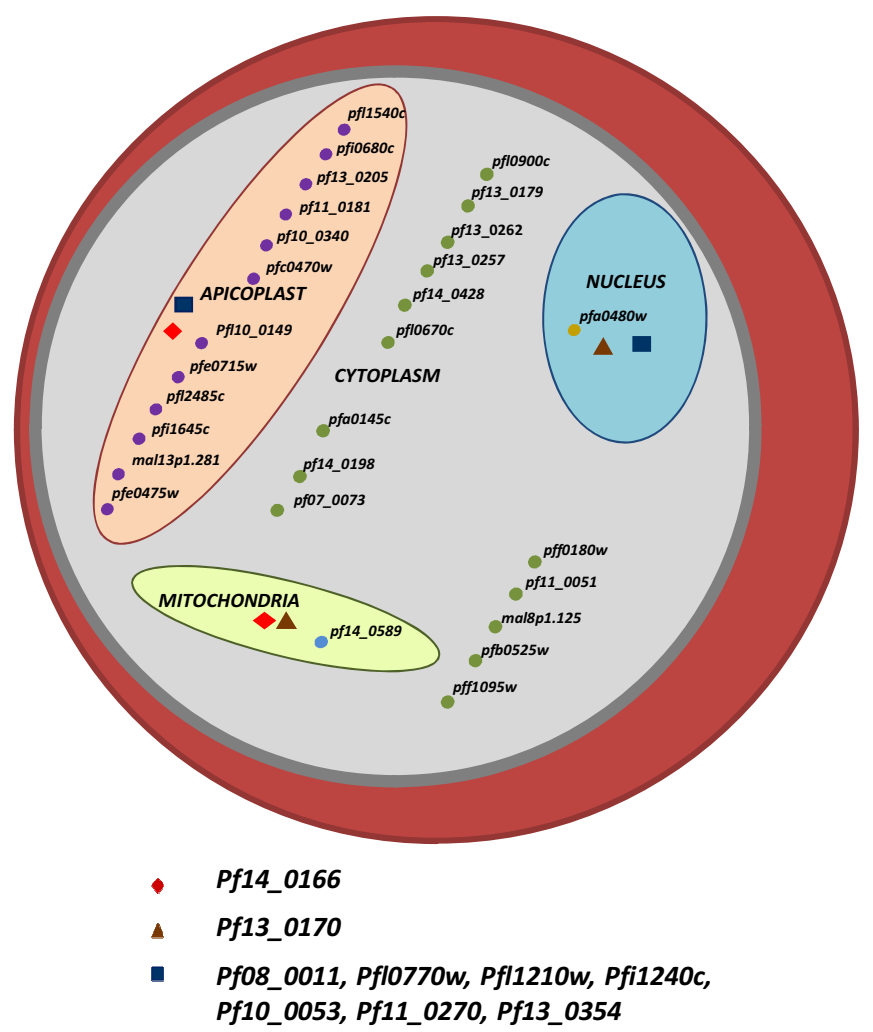

Figure 3

(a) Percentage predicted distribution of Pf-aaRSs in different organelles within the parasite. (b) A schematic of all Pf-aaRSs and their predicted cellular localization. Detailed information regarding gene IDs can be found in additional file I. PfaaRSs predicted to be common between apicoplast \& mitochondria, mitochondria \& nucleus and apicoplast \& nucleus are marked with diamond, triangle and square shapes respectively.

of unusual domain fusions in $P$. falciparum aaRSs suggests multiple functional roles for many of these $P$. falciparum enzymes as has been shown in other organisms.

\section{Phylogenetics}

Overall the percentage identity between matching human and $P$. falciparum aaRS domains varies from 17 to 51 . Clearly, Pf-aaRSs which have low sequence identity with human counterparts might serve as good drug targets. In order to study evolutionary relationships of $P$. falciparum aaRSs with other species, phylogenetic trees were developed in PHYML using maximum likelihood method. For each type of $P$. falciparum aaRS a separate tree was constructed (see additional file 2). aaRS sequences from 102 different species were used for multiple sequence alignments. As an example, phylogenetic tree of TyrRS from various species (including two sequences from $P$. falciparum) was constructed. Interestingly, one Pf-TyrRS
(MAL8P1.125) clustered with human TyrRS whereas the second Pf-TyrRS (PF11_0181) clustered with bacterial TyrRS indicating different evolutionary origins (Figure 6a). Based on distance matrices, several $P$. falciparum aaRS sequences clustered as being closer to plants (A. thaliana) or to bacteria (E. coli) (Figure 6b). It is already known that apicomplexan parasites like $P$. falciparum house a secondary endosymbiotic plastid, possibly hijacked by lateral genetic transfer from an alga. Therefore, the $P$. falciparum aaRS sequences which are evolutionary close to bacteria and plants are likely to be the outcome of horizontal gene transfer from the plastid. $P$. falciparum contains $\sim 12$ such aaRS sequences which cluster with bacterial or plant sequences. Functional and structural characterization of these bacterial/plant-like aaRS may be relevant in focusing efforts at using aaRS as drug targets. 


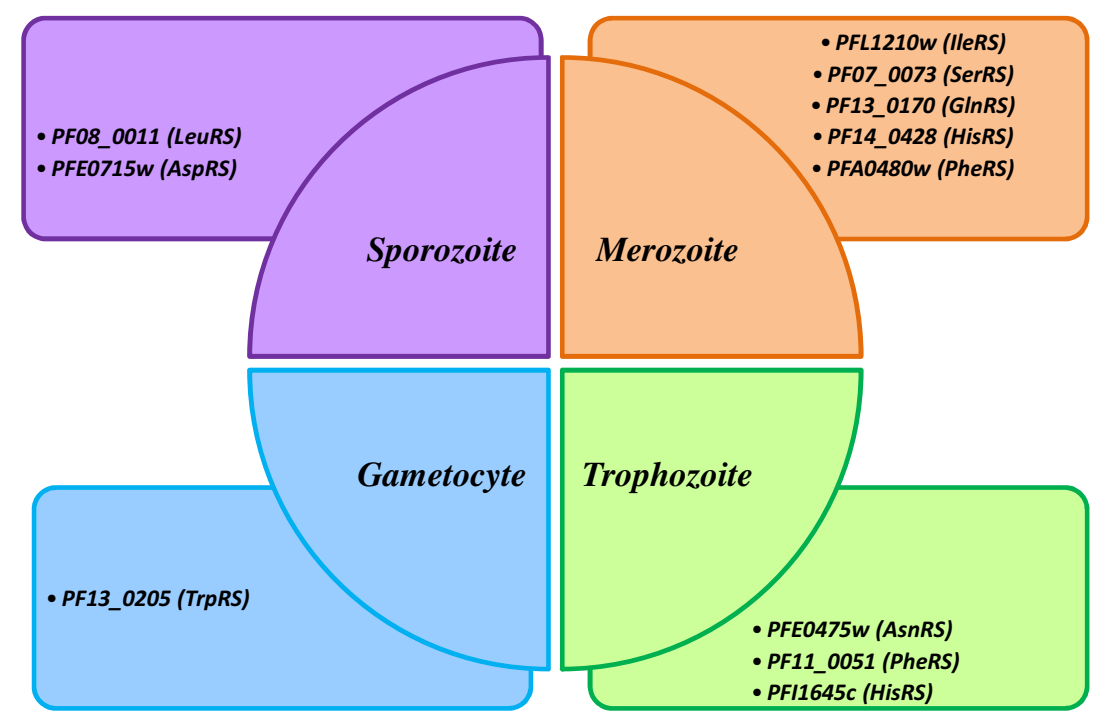

\section{Figure 4}

Diagrammatic representation of Pf-aaRS protein expression which are specifically expressed in different life stages of the parasite based on mass spectrometry data [82].

\section{Homology modeling and structure comparisons}

To date, no crystal structures have been obtained for any aaRS from $P$. falciparum. Hence, we performed homology modeling of several P. falciparum aaRSs using homologous structures available in PDB. Known structural templates ( $\geq 40 \%$ identity) were used for molecular modeling of several $P$. falciparum aaRSs including the two TyrRSs (PF11_0181, MAL8P1.125), the PheRS (PFA0480w), ThrRS (PF11_0270), LysRS (PF13_0262), MetRS (PF10_0340) and TrpRS (PF13_0205). The program Align2D (sequence alignment module in Modeller) was used to perform dynamic programming-based global alignments of the target and template sequences. This program uses variable gap penalty for structural loops and core regions using information derived from template structures. We found key differences in the conserved motifs in various aaRSs. For example, the class I motif 'KYSKS' in P. falciparum TyrRS (PF11_0181) and 'KMSKS' in MAL8P1.125 differs from 'KLGKS' of human mitochondrial TyrRS (2PID) and 'KMSSS' of human cytoplasmic (1N3L) respectively. Similarly, class I motif 'HIGH' has subtle sequences variations between $P$. falciparum and H. sapiens TyrRSs (Figure 7a, Table 3 ). Using the above procedures, we could generate structural models for several Pf-aaRSs. Stereo-chemical qualities of the generated protein models were assessed using PROCHECK (85-90\% residues are in allowed regions of Ramachandran plot). The overall superimposed three-dimension models were visualized in CHIMERA and PYMOL (Figure 7b). Many sequence insertions were observed for $P$. falciparum enzymes when compared to their homologous [63]. Location of insertions in P. falciparum TyrRS between well-con- served secondary structures suggests ability of TyrRS anticodon binding core to accommodate larger sequence inserts with minimum disruption to the catalytic domain. Direct comparison of modeled $P$. falciparum aaRSs with human aaRSs revealed several other important structural differences. For example, numerous insertions are present in the loop regions linking various $\alpha$-helices ( $\alpha 10$ to $\alpha 13$ ) in anticodon binding domain of $P$. falciparum TyrRSs (PF11_0181 and MAL8p1.125) when compared to its human homologous (2PID and 1N3L) respectively. Structural differences between TyrRS (from P. falciparum) and human counterparts are summarized in Table 3 and shown in Figure $7 \mathrm{c}$. These subtle structural changes that manifest as partial conservation of important motifs in $P$. falciparum aaRSs reflect evolutionary divergence, and may be useful for exploitation of parasite-specific features as drug targets.

\section{Conclusion}

Aminoacyl-tRNA synthetases (aaRSs) link RNA with protein translation. Besides their key role in protein synthesis, aaRSs are also integral to various other cellular processes. aaRS enzymes have been the focus for antimicrobial drug discovery $[64,65]$. An example of clinical application of an $a a R S$ inhibitor is provided by the antibiotic mupirocin (marketed as Bactroban), which selectively inactivates bacterial isoleucyl-tRNA synthetase [66]. Similarly, it has been shown that the broad-spectrum antifungal 5-fluoro1,3-dihydro-1-hydroxy-2,1-benzoxaborole (AN2690) inhibits yeast cytoplasmic leucyl-tRNA synthetase by blocking editing site of the enzyme $[67,68]$. Therefore, presence of distinct or tinkered $P$. falciparum aaRS lends an 
Table 2: Transcriptomic and proteomic data for aaRSs in P. falciparum@

\begin{tabular}{|c|c|c|c|c|c|c|c|c|c|c|c|c|}
\hline ID & aaRS & mean I asex & mean2 asex & Gam & spor & TG\$ & $\mathbf{T}$ & Me & G & Sp & Me* & Oocyst/Spor* \\
\hline MALI3PI.28I & AP-GluRs & 4.8 & 10.9 & 39.7 & 6.5 & & & & & & & \\
\hline PF08_00II & AP-LeuRs & 51.4 & 59.1 & 85.4 & 371.6 & & & & & + & & \\
\hline PFI0_0053 & AP-MetRs & 18.7 & 16.5 & 19 & 19 & & & & & & & \\
\hline PFII_0I8I & AP-TyrRs & 13.2 & 2.6 & 0 & 0 & & & & & & & \\
\hline PFI4_0I66 & AP-LysRs & 60.3 & 48.0 & 166.4 & 48.3 & & & & & & & \\
\hline PFE0475w & AP-AsnRs & 104.6 & 92.5 & 222.8 & 149.6 & & + & & & & & \\
\hline PFE07I5w & AP-AspRs & 17.2 & 26.3 & 21.1 & 0 & & & & & + & & \\
\hline PFI0680c & AP-ArgRs & 15.7 & 17.9 & 34.7 & 1.1 & & & & & & & \\
\hline PFII240c & AP-ProRs & 10.8 & 14.0 & 19.9 & 4.2 & & & & & & & \\
\hline PFII645c & AP-HisRs & 27.4 & 21.2 & 8.7 & 15 & & + & & & & & \\
\hline PFL0770w & AP-SerRs & 117.6 & 110.9 & 431.3 & 69 & & & & & & & \\
\hline PFLI2IOw & AP-lleRs & 124.0 & 109.5 & 132.3 & 71 & & & + & & & & \\
\hline PFLI540c & AP-PheRs & 13.7 & 9.7 & 6 & 0.1 & & & & & & & \\
\hline mean & & 44.6 & 41.5 & 91.3 & 58.1 & & & & & & & \\
\hline PFII_0270 & ThrRs & 580.0 & 582.1 & 181.2 & 154.6 & + & + & + & + & + & + & + \\
\hline PFI3_0354 & AlaRs & 123.6 & 129.9 & 75.5 & 27.5 & + & & & & & + & + \\
\hline PFI0_0I49 & CysRs & 138.1 & 115.6 & 29.6 & 44.5 & + & & & & & & + \\
\hline MAL8PI.I25 & TyrRs & 225.6 & 260.0 & 134.3 & 259.2 & + & + & + & + & + & + & + \\
\hline PF07_0073 & SerRs & 190.9 & 193.8 & 364.2 & 22.5 & + & & + & & & & + \\
\hline PFIO_0340 & MetRs & 389.3 & 387.5 & 479.9 & 3.4 & + & + & + & & & + & + \\
\hline PFII_005I & PheRs & 126.2 & 126.5 & 46.9 & 1.6 & + & + & & & & & \\
\hline PFI3_0I70 & GlnRs & 558.8 & 570.8 & 272.3 & 214.7 & + & & + & & & & \\
\hline PFI3_0I79 & lleRs & 192.1 & 188.1 & 152.8 & 0 & + & + & + & + & + & + & + \\
\hline PFI3_0205 & TrpRs & 112.6 & 189.4 & 42.8 & 171.8 & + & & & + & & & \\
\hline PFI3_0257 & GluRs & 330.0 & 334.5 & 205.6 & 139 & + & + & + & + & & + & + \\
\hline PFI3_0262 & LysRs & 1048.8 & 971.6 & 591 & 281.9 & + & + & + & + & + & + & + \\
\hline PFI4_0I98 & GlyRs & 93.4 & 80.1 & 102.4 & 0 & + & + & + & & & + & + \\
\hline PFI4_0428 & HisRs & 35.4 & 51.1 & 0 & 0 & + & & + & & & + & \\
\hline PFI4_0589 & ValRs & 48.0 & 33.2 & 5.8 & 0 & + & & + & & + & + & + \\
\hline PFA0I45c & AspRs & 359.2 & 359.2 & 361 & 21.4 & + & + & + & & & & \\
\hline PFA0480w & PheRs & 49.5 & 20.2 & 28.4 & 10.4 & + & & + & & & + & \\
\hline PFB0525w & AsnRs & 575.3 & 686.7 & 493.1 & 106.2 & + & + & + & + & & + & + \\
\hline PFL0670c & ProRs & 105.4 & 108.8 & 50. & 50 & & + & + & + & + & + & + \\
\hline PFL0900c & ArgRs & 118.3 & 120.8 & 37.1 & 76.2 & + & + & + & & + & + & \\
\hline PFL2485c & TrpRs & 38.2 & 51.1 & 16.5 & 15.9 & & & & & & & \\
\hline Mean & & 259.0 & 264.8 & 174.8 & 73.8 & & & & & & & \\
\hline
\end{tabular}

@ Gene expression data are by Le Roch[52]. Mean I and mean2 asex refer to mean values of mRNA abundance along asexual stages synchronized by sorbitol and by temperature respectively. In the last two columns, mass spectrometry data from purified merozoites (Me*; Leiden Malaria Group, unpublished data) and mosquito stages (Oocyst/Spor*; oocysts, oocystderived sporozoites and salivary gland sporozoites from Anopheles stephensi infected with NF54 strain of $P$. falciparum) are shown.

TG§ proteomic data are obtained by Trophozoites, Gametocytes early and Gametocytes late by Lasonder lab. [88]. T (trophozoites), Me (merozoites), G (gametocytes), Sp (sporozoites) columns refer to data by multi-dimensional protein identification technology in four stages of the parasite life cycle.

opportunity for their exploitation as new drug targets against malaria. In this study, we have extensively analyzed aaRS sequences from Plasmodium species in terms of their mRNA/protein expression profiles, their cellular localization, their organelle targeting and their unique sequence/domain attributes. We have discovered several distinct aaRSs in P. falciparum with no clear human counterparts in terms of their overall domain structures. We have also highlighted deviations of some highly conserved sequence motifs and active site sequence clusters. Our analyses clearly show that a larger fraction of $P$. falci- parum proteome is devoted to aaRS when compared with many other organisms. The phylogenetic data hint at evolutionary closeness of some Pf-aaRSs to bacteria and plants - this further supports the fact of secondary endosymbiosis in this apicomplexan. We hope that our indepth phylogenetic, protein targeting, domain architecture, protein expression profiling and homology modeling data on Pf-aaRSs can be used as a platform for experimental studies of this important protein family in malaria parasites. 


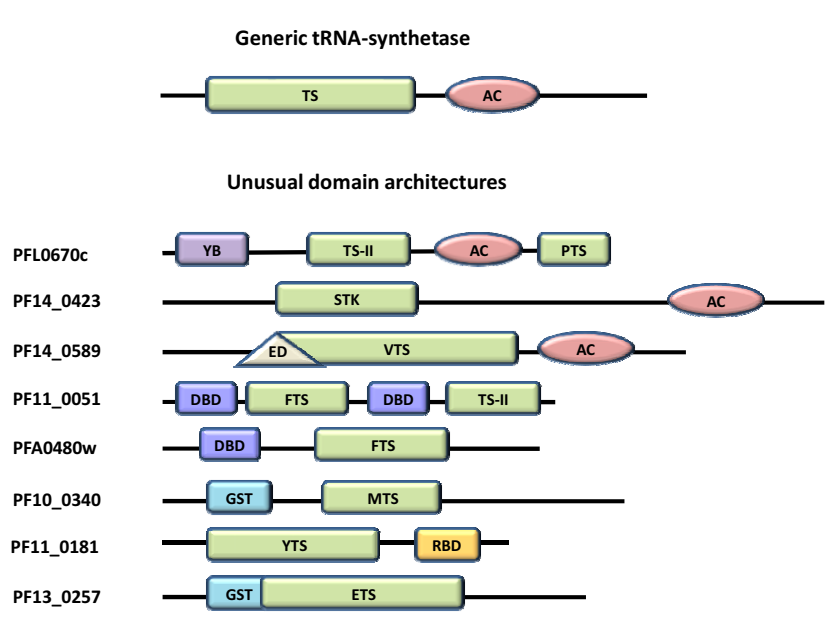

Figure 5

Representation of unusual domain architectures in Pf-aaRSs and related proteins. A generic aaRS is also shown on top. Domain name abbreviations are YB, Ybak associating domain; TS-II, class II tRNA synthetase; AC, anticodon binding site; ED, editing domain; GST, glutathioneStransferase C-terminal region; RBD, S4 RNA binding domain; TS, tRNA synthetase core domain; STK, serine-threonine kinase; FTS, phenylalanine-tRNA synthetase; PTS, prolinetRNA synthetase; VTS, valine-tRNA synthetase; MTS, methionine-tRNA synthetase; YTS, tyrosine-tRNA synthetase; ETS, glutamate-tRNA synthetase.

\section{Methods \\ Sequence extraction}

The P. falciparum genome database PlasmoDB Release 5.4 was used for the present analyses. Sequence sets of all the aaRSs from other organisms includes $P$. berghei, $P$. chabaudi, P. falciparum, P. knowlesi, P. yoelii, P. vivax, H. sapiens, $M$. tuberculosis, D. discoidium, $M$. jannaschii, $R$. norvegicus, $C$. parvum, B. bovis, S. cerevisiae, D. melanogaster, Y. pestis, T. aquaticus, S. pneumoniae, S. entrica, E. coli, A. thatiana, A. pisum, A. salmonicida, B. cereus, B. thuringiensis, B. afzelii, B. burgdorferi, B. garinii, B. valaisiana, Bradyrhizobium, B. pennsylvanicus, C. acidaminovorans, $H$. defensa, C. taiwanensis, E. fergusonii, F. bacterium, F. novicida, F. tularensis, F. alni, G. tenuistipitata, H. arsenicoxydans, A. cellulolyticus, A. chlorophenolicus, A. ferrooxidans, Algoriphagus, A. muciniphila, Anaeromyxobacter, A. thermophilum, B. ambifaria, B. indica, B. mycoides, B. taurus, B. tribocorum, C. atlanticus, Caulobacter, C. aurantiacus, C. cellulolyticum, Citrobacter, C. pinensis, C. Ruthia, Cyanothece, D. desulfuricans, D. hafniense, Diaphorobacter, D. shibae, D. turgidum, E. cuniculi, E. lenta, E. ruminantium, Exiguobacterium, G. diazotrophicus, Geobacillus, M. maris, N. multipartita, Nocardioides, O. terrae, P. abelii, P. atlantica, P. denitrificans, P. ingrahamii, P. lavamentivorans, $R$. castenholzii, S. arenicola, S. fumaroxidans, X. autotrophicus, $V$. vadensis, V. paradoxus, T. whipplei, T. auensis, S. stellata, Ch. parvum, S. heliotrinireducens, Silicibacter, S. putrefaciens, S. usitatus, Thauera, X. laevis, Theileria annulata, Vibrio fischeri, W. succinogenes, $X$. tropicalis, Zeamays. Additional sequences

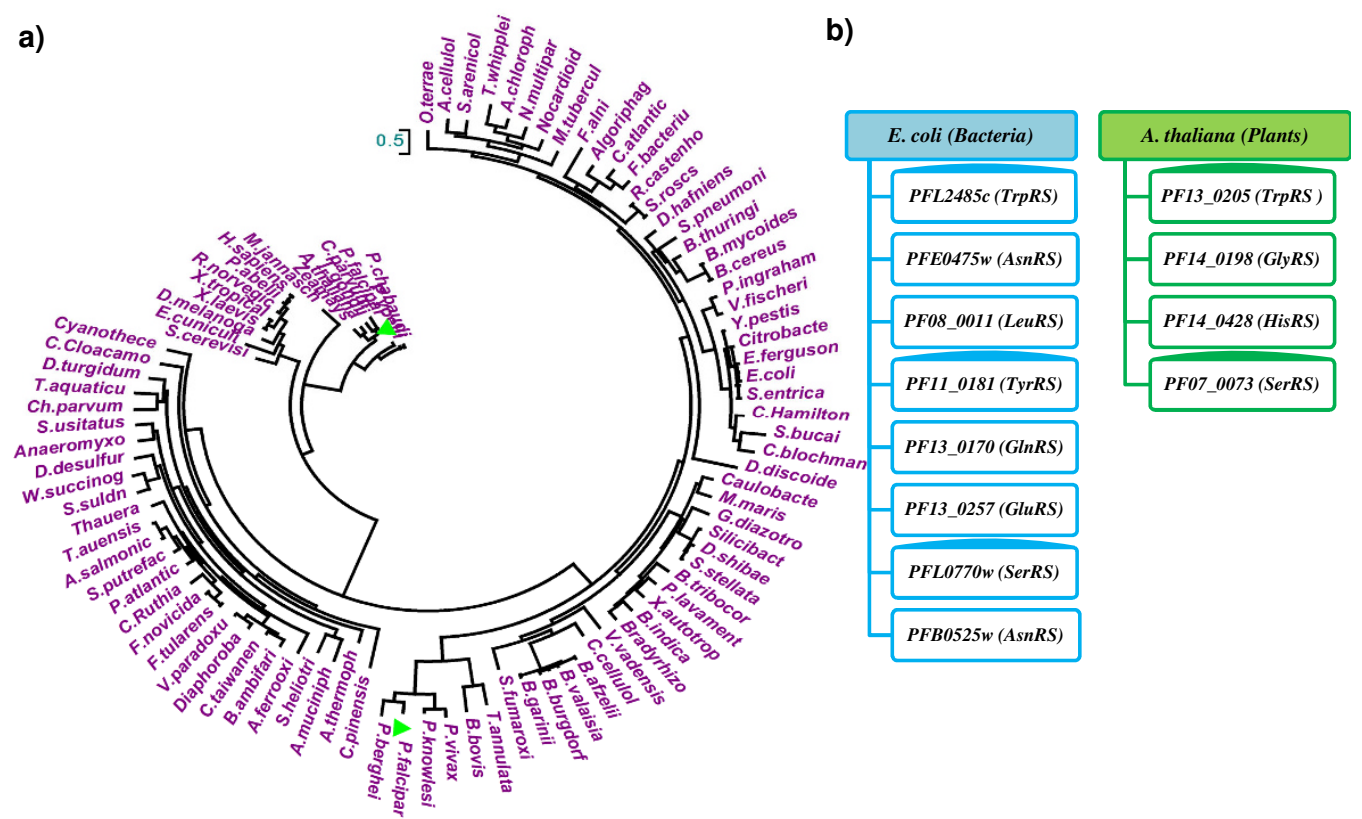

Figure 6

(a) Evolutionary tree was constructed using the PHYML based on maximum likelihood method. P. falciparum TyrRSs (PlasmoDB id -MAL8PI.I25 and PFII_0I8I) are labeled as green triangles. One of the TryRSs (MAL8PI.I25) is evolutionarily closer to H. sapiens whereas the other TyrRS (PFII_0I8I) is closer to E. coli. Total of I02 species were considered for the evolutionary analysis and were taken from three domains of life. (b) List of Pf-aaRS sequences evolutionarily closer to their E. coli and A. Thaliana counterparts. 


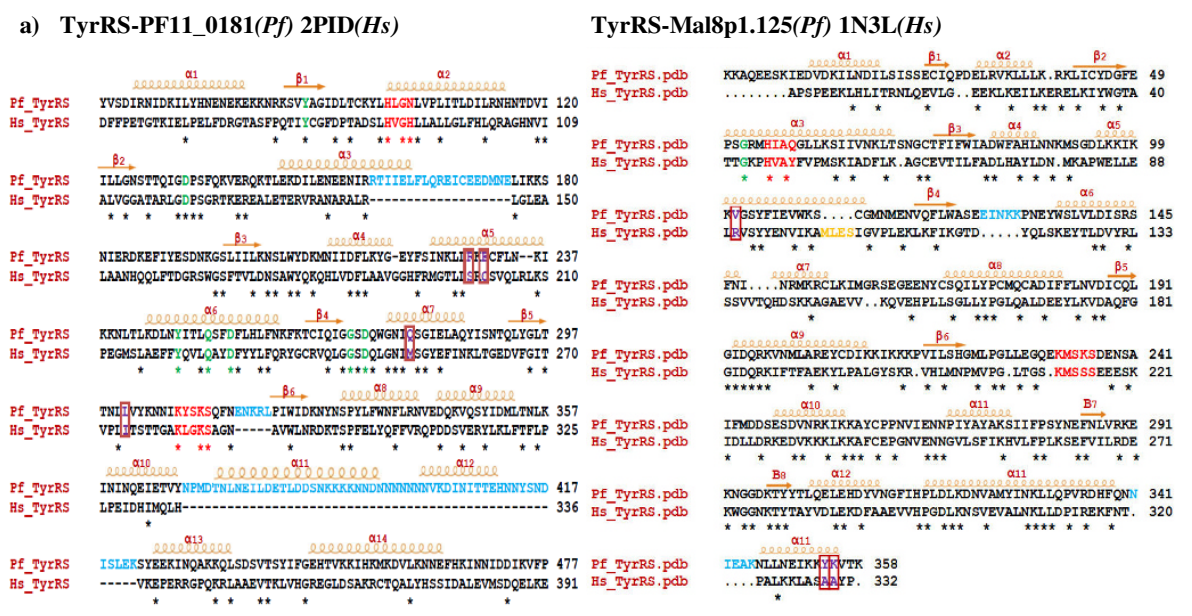

b)
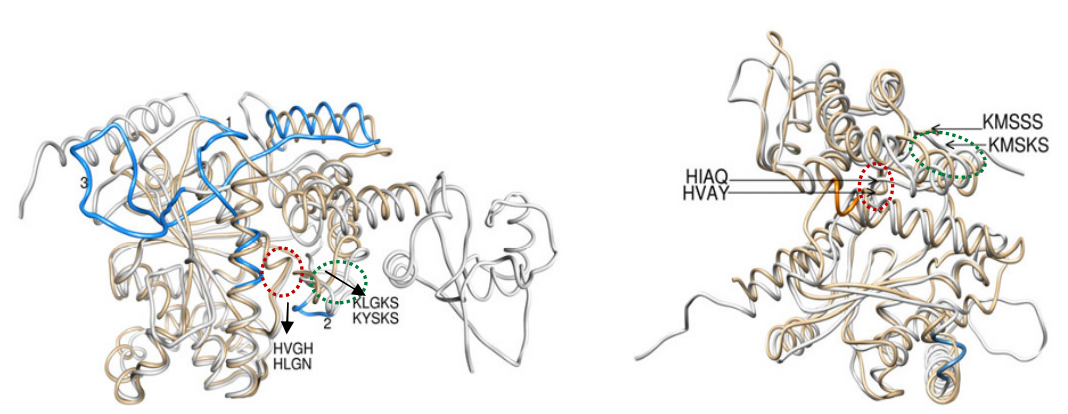

c)
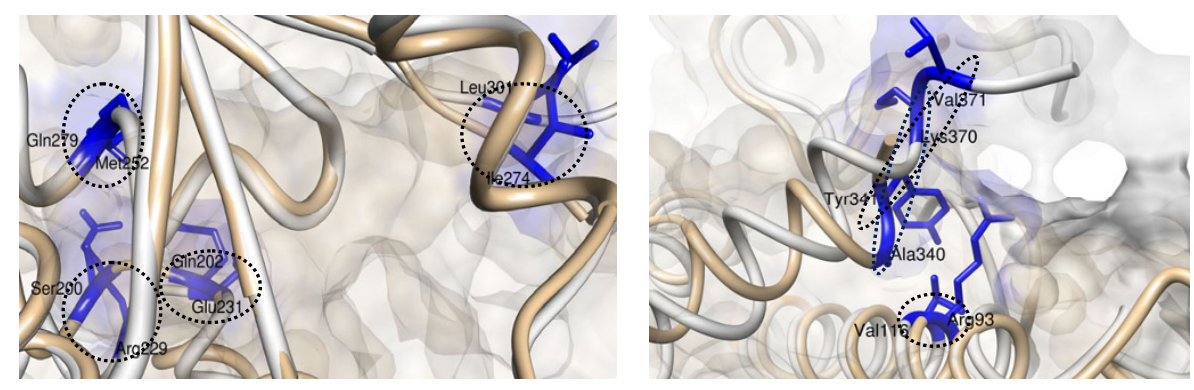

\section{Figure 7}

Left and right panels of the figure represent sequence and structural comparison of bacterial type Plasmodium TyrRS (PFII_0I8I) with human mitochondrial TyrRS (2PID) and the cytosolic Plasmodium TyrRS (Mal8p I.I 25) with human cytosolic TyrRS (IN3L). a) A structure-based sequence alignment of the catalytic domain of Plasmodium TyrRSs with human TyrRSs. Insertions in Pf and human sequences are colored in light blue and orange respectively. Class I synthetase conserved motifs are colored red. Residues involved in tRNA recognition and catalysis are indicated in green (same residues in $\mathrm{Pf}$ and $\mathrm{Hs}$ ) and violet \& boxed (different in $\mathrm{Pf}$ and $\mathrm{Hs}$ ). The secondary structural elements are shown above the sequence alignments. Conserved residues are indicated by asterisk below the sequence alignment. (b) Superposition of $P f-$ TyrRS and Hs-TyrRS depicting the structural differences. Pf-Tyr is colored grey and Hs-TyrRS is colored tan. Insertions in Pf-TyrRSs are highlighted in blue whereas Hs-TyrRS insertions are in orange. Motif I in Pf (PFII_0I8I - HLGN and Mal8pI.I25 - HIAQ) and Hs (2PID - HVGH and IN3L - HVAY) TyrRSs has been encircled red whereas Motif 2 in Pf (PFII_0I8I - KLGKS and Mal8pI.I 25 - KMSKS) and Hs (2PID - KYSKS and IN3L - KMSSS) is encircled green. (c) Snapshot of the active sites of Pf and Hs TyrRSs (superimposed) structures. Non-conserved active site residues colored violet are encircled. 
Table 3: Structural differences between tyrosyl-tRNA synthetases from human \& $P$. falciparum

\begin{tabular}{|c|c|c|c|c|}
\hline & Hs-TyrRS (2PID)* & Pf-TyrRS(PFII_0I8I)@ & Hs-TyrRS (IN3L)! & Pf-TyrRS (MAL8PI.I25)\$ \\
\hline Motif I & $H V G H$ & HLGN & HAVY & HIAQ \\
\hline Motif 2 & KLGKS & KYSKS & KMSSS & KMSKS \\
\hline \multirow{4}{*}{$\begin{array}{c}\text { Residues involved in tyrosine and } \mathrm{A} 73 \\
\text { recognition }\end{array}$} & Ser200 & $\operatorname{Arg} 229$ & Gly46 & Gly67 \\
\hline & $G \ln 202$ & Glu23I & Arg93 & Vall l6 \\
\hline & Met252 & $G \ln 279$ & Ala340 & Lys370 \\
\hline & Ile274 & Leu30I & Tyr34I & Val37I \\
\hline \multirow[t]{3}{*}{ Insertions } & --- & Arg / 57-Glu I 75 & & \\
\hline & --- & Glu3 I6-Leu32 I & Met I04-Ser 107 & Glu I 42-Lys / 46 \\
\hline & ---- & Asn369-Lys 422 & & Asn356-Lys360 \\
\hline
\end{tabular}

\footnotetext{
* human mitochondrial

@ P. falciparum bacteria-like

! human cytosolic

$\$$ P. falciparum human-like
}

were obtained based on sequence similarity via NCBI BLAST [69] and ENSEMBL [70] databases. Known sequence motifs of aaRSs have been used as templates to retrieve sequences of aaRS from other organisms. Some aaRS sequences were manually annotated based on the presence of signature motifs. Protein domains and motifs in the predicted aaRSs were identified using following programs - Superfamily [71], SMART [72] and MotifScan available at expasy web server. The following databases - Pfam [73], TIGR, PIR, EBI and PlasmoDB were also extensively used. Hidden Markov Model (HMM) for each of the 20 aaRS were constructed by the software package Sequence Alignment and Modeling System version 2.2.1 (SAM) [74] exploiting sequences in the aaRS database [75]. HMM profiles were then used to carry out database search $v$ s $P$. falciparum proteins. A score was assigned to each protein by calculating the probability that the corresponding sequence is generated by the HMM model, hence for each database search a score distribution was obtained. The score distributions were normalized and 4 ranges of values were considered to identify aaRS ( $c>5,10<c<20,20<c<50, c<50$ ).

\section{Expression and Localization}

The prediction of signal sequences for cellular localization in $P$. falciparum was performed using various available online web-servers - MITOPROT [76], PredictNLS [77] and PATS [78] for mitochondria, nucleus and apicoplast respectively. PEXEL motif prediction was been carried out by querying PlasmoDB. To identify specific gene expression profiles, we have combined information from different data sets. For the spotted oligonucleotide array data, only half of the 48 time points of the intra-erythrocytic cycle are shown for simplicity, and ratios (versus a common reference) were $\log _{2}$-transformed prior to cluster analysis. For the photolithography data, CEL files were downloaded from website and transferred into Bioconductor package for analysis using a robust multi-array averaging algorithm (RMA) for background adjustment and quantiles normalization [79]. Genes whose expres- sion level was less than 10 (too close to background) or the $\log \mathrm{P}$ was greater than -0.5 (too few probes per gene) were removed from dataset. Total intensity values for each time point were converted to mean-centered ratios by dividing the total intensity by the average intensity for that gene across all experimental conditions and were then $\log _{2}$-transformed prior to clustering. These data manipulations were necessary because the oligo-nucleotide array data was collected as the intensity ratio between the experimental sample and a common reference, while the photolithography data was collected as the total signal intensity at each spot. Gene expression patterns where the minimum percentage of existing values was less than $80 \%$ were eliminated from rest of the analysis. The remaining missing values were replaced by using the $\mathrm{KNN}$-imputation method [80].

\section{Phylogenetic analysis}

To explore the evolutionary relationships amongst aaRSs phylogenetic analyses were performed for each P. falciparum aaRS on an expanded set of 102 sequences. Multiple sequence alignments of these sequences were obtained from CLUSTALW with default parameters (performed locally) in PHYLIP format [81]. These MSAs were used as seed sequences to run PHYML_v2.4.4 using JonesTaylor-Thornton (JTT) model [82]. The resulting file was further used in MEGA4.2 for visualization of trees [83].

\section{Model Building and Validation}

We used Sali's Modeller8v2 [84] tool for building various $P$. falciparum aaRSs models. The stereo-chemical quality of modeled proteins was verified by PROCHECK [85]. Structural mapping of active site residues and other motifs was performed using CHIMERA [86] and PYMOL [87].

\section{Authors' contributions}

TKB, CK and SK carried out the computational experiments and data analysis and wrote the paper; MAJ and VS contributed to the manuscript writing; DS and EP carried 
out HMM construction and database search by HMM; FS performed analysis of transcriptomic and proteomic data; AS designed the study and supervised the work. All authors have read and approved the final manuscript.

\section{Additional material}

\section{Additional file 1}

List of Pf-aaRSs categorized into class I, class II, and related proteins. Gene ID, gene location, description of product and its length are given. Click here for file

[http://www.biomedcentral.com/content/supplementary/14712164-10-644-S1.PDF]

\section{Additional file 2}

Phylogenetic trees of aaRSs from P. falciparum. The evolutionary tree was constructed by the method PHYML using the MEGA 4.0. P. falciparum aaRSs are labeled green triangles. 102 species considered for the evolutionary analysis are taken from the three domains of life viz. P. berghei, P. chabaudi, P. falciparum, P. knowlesi, P. yoelii, P. vivax, H. sapiens, M. tuberculosis, D. discoidium, M. jannaschii, R. norvegicus, C. parvum, B. bovis, S. cerevisiae, D. melanogaster, Y. pestis, T. aquaticus, S. pneumoniae, S. entrica, E. coli, A. thaliana, A. pisum, A. salmonicida, B. cereus, B. thuringiensis, B. afzelii, B. burgdorferi, B. garinii, B. valaisiana, Bradyrhizobium, B. pennsylvanicus, C. acidaminovorans, $\mathrm{H}$. defensa, C. taiwanensis, E. fergusonii, F. bacterium, F. novicida, F. tularensis, F. alni, G. tenuistipitata, H. arsenicoxydans, A. cellulolyticus, A. chlorophenolicus, A. ferrooxidans, Algoriphagus, A. muciniphila, Anaeromyxobacter, A. thermophilum, B. ambifaria, B. indica, B. mycoides, B. taurus, B. tribocorum, C. atlanticus, Caulobacter, C. aurantiacus, C. cellulolyticum, Citrobacter, C. pinensis, C. Ruthia, Cyanothece, D. desulfuricans, D. hafniense, Diaphorobacter, D. shibae, D. turgidum, E. cuniculi, E. lenta, E. ruminantium, Exiguobacterium, G. diazotrophicus, Geobacillus, M. maris, N. multipartita, Nocardioides, O. terrae, P. abelii, P. atlantica, P. denitrificans, P. ingrahamii, P. lavamentivorans, R. castenholzii, S. arenicola, S. fumaroxidans, X. autotrophicus, V. vadensis, V. paradoxus, T. whipplei, T. auensis, S. stellata, Ch. parvum, S. heliotrinireducens, Silicibacter, S. putrefaciens, S. usitatus, Thauera, X. laevis, Theileria annulata, Vibrio fischeri, W. succinogenes, X. tropicalis, Zeamays.

Click here for file

[http://www.biomedcentral.com/content/supplementary/14712164-10-644-S2.PDF]

\section{Acknowledgements}

TKB, CK and AS are supported by grants from the Department of Biotechnology, Govt. Of India. SK is supported by MEPHITIS grant. This work has been conducted as part of MEPHITIS project and partially funded by the European Commission (Grant Agreement no: HEALTH-F3-2009-223024).

\section{References}

I. Ibba M, Soll D: Aminoacyl-tRNA synthesis. Annu Rev Biochem 2000, 69:617-650.

2. Ibba M, Soll $D$ : The renaissance of aminoacyl-tRNA synthesis. EMBO Rep 200I, 2:382-387.

3. Eriani G, Delarue M, Poch O, Gangloff J, Moras D: Partition of tRNA synthetases into two classes based on mutually exclusive sets of sequence motifs. Nature 1990, 347:203-206.
4. Burbaum JJ, Schimmel P: Structural relationships and the classification of aminoacyl- tRNA synthetases. J Biol Chem 1991, 266:16965-16968.

5. Wolf YI, Aravind L, Grishin NV, Koonin EV: Evolution of aminoacyl-tRNA synthetases-analysis of unique domain architectures and phylogenetic trees reveals a complex history of horizontal gene transfer events. Genome Res. 1999, 9(8):689-710.

6. Mirande M: Aminoacyl tRNA synthetase family from prokaryotes and eukaryotes: structural domains and their implications. Prog Nucleic Acid Res Mol Biol I99I, 40:95-I42.

7. Cahuzac B, Berthonneau E, Birlirakis N, Guittet E, Mirande M: A recurrent RNA binding domain is appended to eukaryotic aminoacyl-tRNA synthetases. EMBO J 2000, 19:445-452.

8. Robinson JC, Kerjan P, Mirande M: Macromolecular assemblage of aminoacyl-tRNA synthetases: quantitative analysis of protein-protein interactions and mechanism of complex assembly. J Mol Biol 2000, 304:983-994.

9. Guigou L, Shalak V, Mirande M: The tRNA-interacting factor $p 43$ associates with mammalian arginyl-tRNA synthetase but does not modify its tRNA aminoacylation properties. Biochemistry 2004, 43:4592-4600.

10. Hausmann $C D$, Ibba $M$ : Aminoacyl-tRNA synthetase complexes: molecular multitasking revealed. FEMS Microbiology Reviews 2008, 32:705-72I.

II. Bandyopadhyay AK, Deutscher MP: Complex of aminoacyl-transfer RNA synthetases. J Mol Biol 197।, 60: I 13-122.

12. Kerjan P, Cerini C, Semeriva M, Mirande M: The multienzyme complex containing nine aminoacyl-tRNA synthetases is ubiquitous from Drosophila to mammals. Biochem Biophys Acta 1994, I 1 99:293-297.

13. Wolfson A, Knight R: Occurrence of the aminoacyl-tRNA synthetases in high-molecular weight complexes correlates with the size of substrate amino acids. FEBS Lett 2005, 579:3467-3472.

14. Schimmel P, Schmidt E: Residues in a class I tRNA synthetase which determine selectivity of amino acid recognition in the context of tRNA. Biochemistry 1995, 34: I I 204-I I 2 I0.

15. Lin L, Schimmel P: Mutational analysis suggests the same design for editing activities of two tRNA synthetases. Biochemistry 1996, 35:5596-560I.

16. Sokabe M, Okada A, Yao M, Nakashima T, Tanaka I: Molecular basis of alanine discrimination in editing site. Proc Natl Acad Sci USA 2005, 102: I1669-11674.

17. Sokabe M, Ose T, Nakamura A, Tokunaga K, Nureki O, Yao M, Tanaka I: The structure of alanyl-tRNA synthetase with editing domain. Proc Natl Acad Sci U S A. 2009, 106 (27): I I028-I I 033.

18. Tardif KD, Liu M, Vitseva O, Hou YM, Horowitz J: Misacylation and editing by Escherichia coli valyl-tRNA synthetase: evidence for two tRNA binding sites. Biochemistry 200I, 40:8I I8-8I25.

19. Betha AK, Williams AM, Martinis SA: Isolated CPI domain of Escherichia coli leucyl- tRNA synthetase is dependent on flanking hinge motifs for amino acid editing activity. Biochemistry 2007, 46:6258-6267.

20. Zhao MW, Zhu B, Hao R, Xu MG, Eriani G, Wang ED: Leucyl-tRNA synthetase from the ancestral bacterium Aquifex aeolicus contains relics of synthetase evolution. Embo J 2005, 24:1430-1439.

2I. Ambrogelly A, Ahel I, Polycarpo C: Methanocaldococcus jannaschii prolyl-tRNA synthetase charges tRNAPro with cysteine. J Biol Chem 2002, 277:34749-34754.

22. Ruan B, Söll D: The bacterial YbaK protein is a Cys-tRNAPro and Cys-tRNACys deacylase. J Biol Chem 2005, 280:25887-2589I.

23. Chong YE, Yang XL, Schimmel P: Natural homolog of tRNA synthetase editing domain rescues conditional lethality caused by mistranslation. J Biol Chem 2008, 283:30073-30078.

24. An S, Musier-Forsyth K: Trans-editing of Cys-tRNAPro by Haemophilus influenzae YbaK protein. J Biol Chem 2004, 279:42359-42362.

25. Dwivedi S, Kruparani SP, Sankaranarayanan R: A D-amino acid editing module coupled to the translational apparatus in archaea. Nat Struct Mol Biol 2005, I 2:556-7.

26. Ko YG, Kang YS, Kim EK, Park SG, Kim S: Nucleolar localization of human methionyl-tRNA synthetase and its role in ribosomal RNA synthesis. J Cell Biol 2000, 149:567-574. 
27. Martinis SA, Plateau P, Cavarelli J, Florentz C: Aminoacyl-tRNA synthetases: a family of expanding functions. $E M B O J 1999$, | 8:459|-4596.

28. Cherniack AD, Garriga G, Kittle JD, Akins RA, Lambowitz AM: Function of Neurospora mitochondrial tyrosyl-tRNA synthetase in RNA splicing requires an idiosyncratic domain not found in other synthetases. Cell 1990, 62:745-755.

29. Sampath P, Mazumder B, Seshadri V, Gerber CA, Chavatte L, Kinter M, Ting SM, Dignam JD, Kim S, Driscoll DM, Fox PL: Noncanonical function of glutamyl-prolyl-tRNA synthetase: gene-specific silencing of translation. Cell 2004, I I9: I47-I48.

30. Herzog W, Muller K, Huisken J, Stainier DYR: Genetic evidence for a noncanonical function of seryl-tRNA synthetase in vascular development. Circulation Research 2009, 104:1260-I 266.

3I. Ramya TNC, Karmodiya K, Surolia A, Surolia N: I 5-Deoxyspergualin Primarily Targets the Trafficking of Apicoplast Proteins in Plasmodium falciparum. J Biol Chem 2007, 282:6388-6397.

32. Stoeckert CJ Jr, Fischer S, Kissinger JC, Heiges M, Aurrecoechea C, Gajria B, Roos DS: PlasmoDB v5: new looks, new genomes. Trends Parasitol 2006, 22:543-546.

33. Francklyn C, Perona J], Puetz J, Hou YM: Aminoacyl-tRNA synthetases: Versatile players in the changing theater of translation. RNA 2002, 8:1363-1372.

34. Feng L, Sheppard K, Tumbula-Hansen D, Söll D: GIn-tRNAGln formation from Glu-tRNAGIn requires cooperation of an asparaginase and a Glu-tRNAGIn kinase. I Biol Chem 2005, 280:8I50-8I55.

35. Tumbula DL, Becker HD, Chang WZ, Söll D: Domain-specific recruitment of amide amino acids for protein synthesis. Nature 2000, 407:106-110.

36. Sheppard K, Akochy PM, Salazar JC, Söll D: The Helicobacter pylor amidotransferase GatCAB is equally efficient in glutaminedependent transamidation of Asp-tRNAAsn and Glu-tRNAGln. I Biol Chem 2007, 282: I I866-I I873.

37. Schön A, Kannangara CG, Gough S, Söll D: Protein biosynthesis in organelles requires misaminoacylation of tRNA. Nature 1988 , 331: $187-190$.

38. Jahn D, Kim YC, Ishino Y, Chen MW, Söll D: Purification and functional characterization of the Glu-tRNA(GIn) amidotransferase from Chlamydomonas reinhardtii. I Biol Chem 1990 265:8059-8064.

39. Kim Sl, Stange-Thomann N, Martins O, Hong KW, Söll D, Fox TD: A nuclear genetic lesion affecting Saccharomyces cerevisiae mitochondrial translation is complemented by a homologous Bacillus gene. J Bact 1997, 179:5625-5627.

40. Sheppard K, Söll D: On the evolution of the tRNA-dependent amidotransferases, GatCAB and GatDE. J Mol Biol 2008, 377:831-844.

4I. Hauenstein SI, Perona J]: Redundant synthesis of cysteinyltRNACys in Methanosarcina mazei. I Biol Chem 2008, 283:22007-22017.

42. Sauerwald A, Zhu W, Major TA, Roy H, Palioura S, Jahn D, Whitman WB, Yates JR, lbba M, Söll D: RNA-dependent cysteine biosynthesis in archaea. Science 2005, 307:1969-1972.

43. Fukunaga R, Yokoyama S: Structural insights into the second step of RNA-dependent cysteine biosynthesis in Archaea: crystal structure of Sep-tRNA:Cys-tRNA synthase from Archaeoglobus fulgidus. J Mol Biol 2007, 370: I28-141.

44. Quevillon S, Agou F, Robinson JC, Mirande M: The p43 component of the mammalian multi-synthetase complex is likely to be the precursor of the endothelial monocyte-activating polypeptide II cytokine. I Biol Chem 1997, 272:32573-32579.

45. Behrensdorf HA, van de Craen M, Knies UE, Vandenabeele $P$, Clauss M: The endothelial monocyte-activating polypeptide II (EMAP II) is a substrate for caspase-7. FEBS Lett 2000, 466: $143-147$.

46. Faisal W, Symonds P, Panjwani S, Heng Y, Murray JC: Cell-surface associated p43/endothelial-monocyte-activating-polypeptide-II in hepatocellular carcinoma cells induces apoptosis in T-lymphocytes. Asian J Surg 2007, 30: I 3-22.

47. Shalak V, Kaminska M, Mitnacht-Kraus R, Vandenabeele P, Clauss M, Mirande M: The EMAPII cytokine is released from the mammalian multisynthetase complex after cleavage of its p43/ proEMAPII component. J Biol Chem 200I, 276:23769-23776.

48. Hopkins J, Fowler R, Krishna S, Wilson I, Mitchell G, Bannister L: The plastid in Plasmodium falciparum asexual blood stages: a three-dimensional ultrastructural analysis. Protist 1999 , 150:283-295.

49. Lund E, Dahlberg JE: Proofreading and aminoacylation of tRNAs before export from the nucleus. Science 1998 282:2082-2085

50. Cooke BM, Lingelbach K, Bannister LH, Tilley L: Protein trafficking in Plasmodium falciparum -infected red blood cells. Trends Parasitol 2004, 20:58I-589.

5I. Hiller NL, Bhattacharjee S, van Ooij C, Liolios K, Harrison T: A hosttargeting signal in virulence proteins reveals a secretome in malarial infection. Science 2004, 306: 1934-1937.

52. Le Roch KG, Zhou Y, Blair PL, Grainger M, Moch JK, Haynes JD, De La, Vega P, Holder AA, Batalov S, Carucci DJ, Winzeler EA: Discovery of gene function by expression profiling of the malaria parasite life cycle. Science 2003, 30 I:| 487-|488.

53. Bozdech Z, Llinas M, Pulliam BL, Wong ED, Zhu J, DeRisi JL: The transcriptome of the intraerythrocytic developmental cycle of Plasmodium falciparum. PLoS Biol 2003, I:e5.

54. O'Donoghue P, Luthey-Schulten Z: Evolution of Structure in Aminoacyl-tRNA synthetases. Microbiology and Molecular Biology Reviews 2003, 67:550-573.

55. Salazar JC, Ambrogelly A, Crain PF, McCloskey JA, Söll D: A truncated aminoacyl-tRNA synthetase modifies RNA. Proc Nat Acad Sci USA 2004, I 01:7536-754I.

56. An S, Musier-Forsyth K: Cys-tRNA(Pro) editing by Haemophilus influenzae YbaK via a novel synthetase.YbaK.tRNA ternary complex. J Biol Chem 2005, 280:34465-72.

57. Dou X, Limmer S, Kreutzer R: DNA-binding of phenylalanyltRNA synthetase is accompanied by loop formation of the double-stranded DNA. Journal of Molecular Biology 200I, 305:45I-458

58. Seror SJ, Casaregola S, Vannier F, Zoauri N, Dahl M, Boye E: A mutant cysteinyl-tRNA synthetase affecting timing of chromosomal replication initiation in $B$. subtilis and conferring resistance to a protein kinase C inhibitor. EMBO J 1994, 13:2472-2480.

59. Kim JY, Kang YS, Lee JW, Kim HJ, Ahn YH, Park H, Ko YG, Kim S: p38 is essential for the assembly and stability of macromolecular tRNA synthetase complex: implications for its physiological significance. Proc Natl Acad Sci USA 2002, 99:79|2-79|6.

60. Bec G, Kerjan P, Zha XD, Waller JP: Valyl-tRNA synthetase from rabbit liver. I. Purification as a heterotypic complex in association with elongation factor I. J Biol Chem 1989 , 264:2I|3|-2|I37.

61. Bec G, Kerian P, Waller JP: Reconstitution in vitro of the valyltRNA synthetase-elongation factor (EF) I beta gamma delta complex. Essential roles of the NH2- terminal extension of valyl-tRNA synthetase and of the EF-I delta subunit in complex formation. J Biol Chem 1994, 269:2086-2092.

62. Brandsma M, Kerjan P, Dijik J, Janssen GM, Moller W: Valyl-tRNA synthetase from Artemia. Purification and association with elongation factor I. Eur J Biochem 1995, 233:277-282.

63. Kumar R, Musiyenko A, Oldenburg A, Adams B, Barik S: Post-translational generation of constitutively active cores from larger phosphatases in the malaria parasite, Plasmodium falciparum: implications for proteomics. BMC Mol Biol 2004, 5:6.

64. He CY, Shaw MK, Pletcher CH, Striepen B, Tilney LG, Roos DS: A plastid segregation defect in the protozoan parasite Toxoplasma gondii. EMBO / 200I, 20:330-339.

65. Waller RF, McFadden Gl: The apicoplast: a review of the derived plastid of apicomplexan parasites. Curr Issues Mol Biol 2005, 7:57-79.

66. Fichera ME, Roos DS: A plastid organelle as a drug target in apicomplexan parasites. Nature 1997, 390:407-409.

67. Boyce JM: MRSA patients: proven methods to treat colonization and infection. J Hosp Infect 200I, 48:S9-SI4.

68. Rock FL, Mao W, Yaremchuk A, Tukalo M, Crépin T, Zhou H, Zhan YK, Hernandez V, Akama T, Baker SJ, Plattner JJ, Shapiro L, Martinis SA, Benkovic S], Cusack S, Alley MRK: An Antifungal Agent Inhibits an Aminoacyl-tRNA Synthetase by Trapping tRNA in the Editing Site. Science 2007, 316:1759-176|.

69. Altschul SF, Madden TL, Schaffer AA, Zhang J, Zhang Z, Miller W, Lipman DJ: Gapped BLAST and PSI-BLAST: a new generation of protein database search programs. Nucleic Acids Res 1997, 25:3389-3402. 
70. Stalker J, Gibbins B, Meidl P, Smith J, Spooner W, Hotz HR, Cox AV: The Ensembl Web Site: Mechanics of a Genome Browser. Genome Res 2004, I4:95I-955.

7I. Wilson D, Pethica R, Zhou Y, Talbot C, Vogel C, Madera M, Chothia C, Gough J: SUPERFAMILY - Comparative Genomics, Datamining and Sophisticated Visualisation. Nucleic Acids Res 2009, 37:380-386.

72. Letunic I, Doerks T, Bork P: SMART 6: Recent updates and new developments. Nucleic Acids Res 2008, 37:229-232.

73. Bateman A, Birney E, Cerruti L, Durbin R, Etwiller L, Eddy SR, Griffiths-Jones S, Howe KL, Marshall M, Sonnhammer EL: The Pfam protein families database. Nucleic Acids Res 2002, 30:276-280.

74. Hughey R, Krogh A: Hidden Markov models for sequences analysis: Extension and analysis of the basic method. Computer Applications in the Biosciences 1996, I 2:95-107.

75. Szymanski M, Deniziak MA, Barciszewski J: Aminoacy-tRNA synthetases database. Nucleic Acids Res 200I, 29:288-290.

76. Claros MG, Vincens P: Computational method to predict mitochondrially imported proteins and their targeting sequences. Eur / Biochem 1996, 24 I:779-786.

77. Cokol M, Nair R, Rost B: Finding nuclear localization signals. EMBO reports 2000, I:4II -4I 5 .

78. Zuegge J, Ralph S, Schmuker M, McFadden GI, Schneider G: Deciphering apicoplast targeting signals - feature extraction from nuclear-encoded precursors of Plasmodium falciparum apicoplast proteins. Gene 200I, 280:19-26.

79. Irirzarry RA, Hobbs B, Collin F, Beazer-Barclay YD, Antonellis KJ, Scherf U, Speed TP: Exploration, normalization and summaries of high densities of oligonucleotide array probe level data. Biostatistics 2003, 4:249-264.

80. Troyanskaya O, Cantor M, Sherlock G: Missing value estimation method for DNA microarrays. Bioinformatics 200I, 17:520-525.

81. Thompson JD, Higgins DG, Gibson TJ: CLUSTAL W: improving the sensitivity of progressive multiple sequence alignment through sequence weighting, position-specific gap penalties and weight matrix choice. Nucleic Acids Res 1994, 22:4673-4680.

82. Stéphane G, Franck L, Patrice D, Olivier G: PHYML Online--a web server for fast maximum likelihood-based phylogenetic inference. Nucleic Acids Res 2005:W557-W559.

83. Kumar S, Dudley J, Nei M, Tamura K: MEGA: A biologist-centric software for evolutionary analysis of DNA and protein sequences. Briefings in Bioinformatics 2008, 9:299-306.

84. Renom MA, Stuart A, Fiser A, Sánchez R, Melo F, Sali A: Comparative protein structure modeling of genes and genomes. Annu Rev Biophys Biomol Struct 2000, 29:291-325.

85. Laskowski RA, MacArthur MW, Moss DS, Thornton JM: PROCHECK: a program to check the stereochemical quality of protein structures. J Appl Cryst 1993, 26:283-291.

86. Pettersen EF, Goddard TD, Huang CC, Couch GS, Greenblatt DM, Meng EC, Ferrin TE: UCSF Chimera - A Visualization System for Exploratory Research and Analysis. J Comput Chem 2004, 25:1605-1612.

87. DeLano WL: The PyMOL Molecular Graphics System. DeLano Scientific, San Carlos, CA, USA 2002 [http://www.pymol.org].

88. Lasonder E, Ishihama $Y$, Andersen JS, Vermunt AM, Pain A, Sauerwein RW, Eling WM, Hall N, Waters AP, Stunnenberg HG, Mann M: Analysis of the Plasmodium falciparum proteome by high-accuracy mass spectrometry. Nature 2002, 41 9:537-542.

Publish with Biomed Central and every scientist can read your work free of charge

"BioMed Central will be the most significant development for disseminating the results of biomedical research in our lifetime. "

Sir Paul Nurse, Cancer Research UK

Your research papers will be:

- available free of charge to the entire biomedical community

- peer reviewed and published immediately upon acceptance

- cited in PubMed and archived on PubMed Central

- yours - you keep the copyright
BioMedcentral 